\title{
CONTINUITY PROPERTIES OF MONOTONE NONLINEAR OPERATORS IN BANACH SPACES
}

\author{
BY FELIX E. BROWDER ${ }^{1}$ \\ Communicated March 12, 1964
}

In a number of recent papers, the writer $([1]-[11])$ and G. J. Minty ([13]-[16]) have studied nonlinear functional equations in Banach spaces involving monotone operators satisfying various mild continuity conditions (in particular demicontinuity, hemicontinuity, and vague continuity). In his note [12], T. Kato has studied the inter-relations of these continuity assumptions for monotone operators and shown in particular that every hemicontinuous locally bounded monotone operator $G$ from a Banach space $X$ to its dual $X^{*}$ is always demicontinuous. The writer has independently obtained this and related results by a slightly different method in connection with his study of multi-valued monotone nonlinear mappings [9]. We present this argument below in $\$ 1$.

1. Let $X$ be a complex Banach space, $X^{*}$ the space of bounded conjugate-linear functionals on $X,(w, u)$ the pairing between $w$ in $X^{*}$ and $u$ in $X$. Following the notation of [12], $\rightarrow$ denotes strong convergence in $X$ or $X^{*}, \rightarrow$ weak $^{*}$ convergence in $X^{*}$.

Let $G$ be a function with domain $D=D(G) \subset X$ and values in $X^{*}$. Then:

(1) $G$ is said to be monotone if

$$
\operatorname{Re}(G u-G v, u-v) \geqq 0
$$

for all $u, v$ in $D$.

(2) $G$ is said to be demicontinuous if $u_{n} \rightarrow u$ in $D$ implies $G u_{n} \rightarrow G u$.

(3) $G$ is said to be hemicontinuous if $u \in D, v \in X$ and $u+t_{n} v \in D$ where $t_{n}>0, t_{n} \rightarrow 0$, together imply $G\left(u+t_{n} v\right) \rightarrow G u$.

(4) $G$ is said to be vaguely continuous if $u \in D, v \in X$ and $u+t v \in D$ for $0<t<t_{0}$ for some $t_{0}>0$ imply that there exists a sequence $\left\{t_{n}\right\}$ with $t_{n}>0$ for all $n, t_{n} \rightarrow 0$ as $n \rightarrow+\infty$ such that $G\left(u+t_{n} v\right) \rightarrow G u$.

(5) $G$ is said to be $D$-maximal monotone if for $u_{0} \in D$, $w_{0} \in X^{*}$, the inequality $\operatorname{Re}\left(w_{0}-G u, u_{0}-u\right) \geqq 0$ for all $u$ in $D$ implies that $w_{0}$ $=G\left(u_{0}\right)$.

(6) $G$ is said to be locally bounded if for any sequence $\left\{u_{n}\right\}$ in $D$

1 This research was supported in part by National Science Foundation grants NSF-G19751 and NSF-GP2283, and in part by Army Research Office (Durham) grant ARO(D)-31-124-G455. 
with $u_{n} \rightarrow u$ where $u \in D$, we have $G u_{n}$ bounded.

(7) $D$ is said to be quasi-dense [12] if for each $u$ in $D$ there exists a dense subset $M_{u}$ of $X$ such that for each $v \in M_{u}, u+t v \in D$ for sufficiently small $t>0$ (the smallness of $t$ depending on $v$ ).

Theorem 1. If $G$ is D-maximal monotone and locally bounded, then $G$ is demicontinuous.

Proof of Theorem 1. Let $\left\{u_{n}\right\}$ be a sequence in $D$ with $u_{n} \rightarrow u_{0}$, $u_{0} \in D$. Since $\left\{G u_{n}\right\}$ is bounded in $X^{*}$, to show that $G u_{n} \rightarrow G u_{0}$, it suffices to show that if $G u_{n} \rightarrow w_{0}$, then $w_{0}=G u_{0}$. Let $u$ be any element of $D$. For each $n, \operatorname{Re}\left(G u_{n}-G u, u_{n}-u\right) \geqq 0$. As $n \rightarrow \infty, u_{n}-u \rightarrow 0$, $G u_{n}-G u \rightarrow w_{0}-G_{u}$. Hence

$$
\operatorname{Re}\left(w_{0}-G u, u_{0}-u\right) \geqq 0 .
$$

Since $G$ is $D$-maximal monotone, $w_{0}=G u_{0}$. Q.E.D.

Theorem 2. Suppose that $G$ is monotone and vaguely continuous and that its domain $D$ is quasi-dense. Then $G$ is D-maximal monotone.

Proof of Theorem 2. Suppose $w_{0} \neq G u_{0}$. Since $D$ is quasi-finitely dense, there exists $v$ in $M_{u_{0}}$ such that $u_{0}+t v$ lies in $D$ for $0<t<t_{0}(v)$ while $\operatorname{Re}\left(w_{0}-G u_{0}, v\right)>0$.

By the vague continuity of $G$, we may find a sequence of positive numbers $\left\{t_{n}\right\}$ with $t_{n} \rightarrow 0$ as $n \rightarrow \infty$ such that $G\left(u_{0}+t_{n} v\right) \rightarrow G u_{0}$. Then for every $n$ :

$$
0 \leqq \operatorname{Re}\left(G\left(u_{0}+t_{n} v\right)-w_{0}, t_{n} v\right)
$$

so that since $t_{n}>0$,

$$
0 \leqq \operatorname{Re}\left(G\left(u_{0}+t_{n} v\right)-w_{0}, v\right) .
$$

Hence

$$
0<\operatorname{Re}\left(w_{0}-G u_{0}, v\right) \leqq \lim \inf \operatorname{Re}\left(G\left(u_{0}+t_{n} v\right)-G u_{0}, v\right)=0,
$$

which is a contradiction. Q.E.D.

Theorem 3. Suppose that $G$ is monotone, locally bounded, and vaguely continuous while $D$ is quasi-dense. Then $G$ is demicontinuous.

Theorem 3 is a corollary of Theorems 1 and 2 and contains Theorem 1 of [12] as a special case.

REMARK. In Theorem 2 of [12], Kato shows that for finite-dimensional $X$, local boundedness can be eliminated from the hypothesis of the result corresponding to Theorem 3 . This is obviously not the case in infinite dimensional spaces, as one can see from the consideration 
of unbounded non-negative self-adjoint operators in an infinite dimensional Hilbert space. Whether it is true under the additional hypothesis that $D=D(G)=X$ is an open question. We note that in this case it follows from the argument of Theorem 1 above and a category argument that the set of points in $X$ at which $G$ is continuous includes an open dense set.

REMARK 2. The proof given by Kato for his Theorem 2 in [12] actually proves the following:

Theorem 4. If $X$ is of finite dimension, $G$ a monotone mapping of $X$ into $X^{*}$ with quasi-dense domain $D$, then $G$ is locally bounded.

Combining this result with Theorem 3 above, we have:

Theorem 5. Let $X$ be of finite dimension, $G$ a vaguely continuous monotone mapping from $X$ to $X^{*}$ with quasi-dense domain $D$. Then $G$ is continuous.

\section{BIBLIOGRAPHY}

1. F. E. Browder, The solvability of non-linear functional equations, Duke Math. J. 30 (1963), 557-566.

2. - Variational boundary-value problems for quasi-linear elliptic equations. I, II, III, Proc. Nat. Acad. Sci. U.S.A. 30 (1963), 31-37, 592-598, 794-798.

3. - Non-linear elliptic boundary value problems, Bull. Amer. Math. Soc. 69 (1963), 862-874.

4. - Non-linear parabolic boundary value problems of arbitrary order, Bull. Amer. Math. Soc. 69 (1963), 858-861.

5. - Strongly non-linear parabolic boundary value problems, Amer. J. Math. (1964) (to appear).

6. - Non-linear elliptic boundary value problems. II, Trans. Amer. Math. Soc. (to appear).

7. - Non-linear equations of evolution, Ann. of Math. (to appear)

8. - On a theorem of Beurling and Livingston, Canad. J. Math. (to appear).

9. - Multi-valued monotone non-linear mappings and duality mappings in Banach spaces, Trans. Amer. Math. Soc. (to appear).

10. - Non-linear initial value problems (to appear).

11. — - Non-linear elliptic problems. II, Bull. Amer. Math. Soc. 70 (1964), 299-302.

12. T. Kato, Demicontinuity, hemicontinuity, and monotonicity, Bull. Amer. Math. Soc. 70 (1964), 548-550.

13. G. J. Minty, Monotone (nonlinear) operators in Hilbert space, Duke Math. J. 29 (1962), 341-346.

14. - Two theorems on nonlinear functional equations in Hilbert space, Bull. Amer. Math. Soc. 69 (1963), 691-692.

15. - On a "monotonicity" method for the solution of nonlinear equations in Banach spaces, Proc. Nat. Acad. Sci. U.S.A. 50 (1963), 1038-1041.

16. - Maximal monotone sets in Hilbert spaces (to appear).

Institute for Advanced STUdy AND University of Chicago 